\title{
A polyphasic approach for characterization of a collection of cereal isolates of the Fusarium incarnatum-equiseti species complex
}

\author{
Alessandra Villani ${ }^{\mathrm{a}}$, Antonio Moretti ${ }^{\mathrm{a}}$, Sarah De Saeger ${ }^{\mathrm{b}}$, Zheng Han ${ }^{\mathrm{b}}$, Jose Diana Di Mavungu ${ }^{\mathrm{b}}$, \\ Célia M.G. Soares ${ }^{c}$, Robert H. Proctor ${ }^{\mathrm{d}}$, Armando Venâncio ${ }^{\mathrm{c}}$, Nelson Lima ${ }^{\mathrm{c}}$, Gaetano Stea ${ }^{\mathrm{a}}$, Costantino Paciolla ${ }^{\mathrm{e}}$, \\ Antonio F. Logrieco ${ }^{a}$, Antonia Susca ${ }^{\mathrm{a}, *}$ \\ a Institute of Sciences of Food Production, National Research Council, Via Amendola, 122/0, 70126 Bari, Italy \\ b Laboratory of Food Analysis, Department of Bioanalysis, Faculty of Pharmaceutical Sciences, Ghent University, Ottergemsesteenweg 460,9000 Ghent, Belgium \\ c CEB-Centre of Biological Engineering, University of Minho, Campus de Gualtar, 4710, -057 Braga, Portugal \\ d National Center for Agricultural Utilization Research, U.S. Department of Agriculture, 1815 N. University St., Peoria, IL 61604, USA \\ e Department of Biology, University of Bari, Via E. Orabona 4, 70125, Bari, Italy
}

\section{A R T I C L E I N F O}

\section{Article history:}

Received 22 January 2016

Received in revised form 18 April 2016

Accepted 20 June 2016

Available online 22 June 2016

\section{Keywords:}

FIESC

Secondary metabolites

Phylogeny

TRI genes

\begin{abstract}
A B S T R A C T
DNA-based phylogenetic analyses have resolved the fungal genus Fusarium into multiple species complexes. The F. incarnatum-equiseti species complex (FIESC) includes fusaria associated with several diseases of agriculturally important crops, including cereals. Although members of FIESC are considered to be only moderately aggressive, they are able to produce a diversity of mycotoxins, including trichothecenes, which can accumulate to harmful levels in cereals. High levels of cryptic speciation have been detected within the FIESC. As a result, it is often necessary to use approaches other than morphological characterization to distinguish species. In the current study, we used a polyphasic approach to characterize a collection of 69 FIESC isolates recovered from cereals in Europe, Turkey, and North America. In a species phylogeny inferred from nucleotide sequences from four housekeeping genes, 65 of the isolates were resolved within the Equiseti clade of the FIESC, and four isolates were resolved within the Incarnatum clade. Seven isolates were resolved as a genealogically exclusive lineage, designated here as FIESC 31. Phylogenies based on nucleotide sequences of trichothecene biosynthetic genes and MALDI-TOF MS (Matrix-Assisted Laser Desorption/Ionization Time-Of-Flight Mass Spectrometry) were largely concordant with phylogeny inferred from the housekeeping gene. Finally, Liquid Chromatography (Time-Of-Flight) Mass Spectrometry [LC-(TOF-)MS(/MS)] revealed variability in mycotoxin production profiles among the different phylogenetic species investigated in this study.
\end{abstract}

(c) 2016 Elsevier B.V. All rights reserved.

\section{Introduction}

Cereals contribute to approximately $30 \%$ of the human diet in industrialized countries (FAOStat, 2013) as well as roughly $50 \%$ of animal feed in Europe (European Commission, Agricultural and Rural Development, Short-term outlook , 2015). Therefore, problems related to cereal crops can be of economic and public health concern. Several pathogenic fungi threaten yield and quality of cereals worldwide due to their ability to cause disease and produce mycotoxins, secondary metabolites that are toxic to humans and other animals (Rocha et al., 2005; Ma et al., 2013; Moretti et al., 2013). The genus Fusarium includes some of the cereal-infecting fungi that are of greatest concern to agricultural production and food/feed safety worldwide (Desjardins, 2006). Collectively, Fusarium species cause multiple diseases that reduce yield and quality, and they

\footnotetext{
* Corresponding author.

E-mail address: antonella.susca@ispa.cnr.it (A. Susca).
}

produce several mycotoxins, including fumonisins, trichothecenes and zearalenone. Trichothecenes (TRI) are among the most economically significant mycotoxins worldwide, and are of particular concern on barley, maize and wheat (Maier et al., 2006; McCormick, 2013). These terpene-derived mycotoxins are strong inhibitors of eukaryotic protein biosynthesis, which in turn can cause a wide range of toxic effects, including vomiting, hemorrhaging and immunosuppression (Desjardins, 2006). Over 200 trichothecene analogs have been described (Grovey, 2007), and can be subdivided into four classes (A, B, C, and D) based on variation in chemical structure. Only type $A$ and type $B$ trichothecenes are produced by Fusarium (Desjardins, 2006). Type B trichothecenes are distinguished by the presence of a keto group at the carbon eight position (C8), and include deoxynivalenol (DON), its acetylated derivatives 3-acetyldeoxynivalenol (3-ADON) and 15acetyldeoxynivalenol (15-ADON), as well as nivalenol (NIV) and its acetylated derivative 4-acetylnivalenol/fusarenone X (4-ANIV/FUS-X). The health risks for humans and other animals associated with exposure 
to trichothecenes are widely recognized, and levels for DON in grains and cereal-based products are regulated by the European Commission (EC No. 1881/2006). Type A trichothecenes have hydrogen (4.15diacetoxyscirpenol), hydroxyl (neosolaniol), or ester moiety (T-2 toxin) at C-8, and they are generally more toxic to animals than type B trichothecenes. Some type A compounds, such as 4.15diacetoxyscirpenol (DAS) and T-2 toxin, are also included on the National Select Agent list in the US (http://www.selectagents.gov).

Within the genus Fusarium, F. culmorum, F. graminearum, F. poae, F. sporotrichioides, and members of $F$. incarnatum-equiseti species complex (FIESC) are reported to produce trichothecenes (Desjardins, 2006). Although members of the FIESC are rarely associated with major disease epidemics, they have been identified as co-occurring with plant and human infections. Members of the FIESC are able to produce not only trichothecenes (both type A and type B), but also a number of other secondary metabolites, such as butenolide, beauvericin, equisetin, fusarochromanone and zearalenone (Desjardins, 2006). Recently, O'Donnell et al. (2009, 2010, 2012) demonstrated by multilocus GCPSR (Genealogical Concordance Phylogenetic Species Recognition) analysis that the FIESC comprises a large number of cryptic species (i.e., that cannot be distinguished by traditional morphology-based identification methods). Evolutionary analysis through Multi-Locus Sequence Typing (MLST), based on four genes sequences (O'Donnell et al., 2009, 2012), has facilitated recognition of 30 phylogenetic species (FIESC 1 through FIESC 30 ) within the FIESC. Some of these species contain multiple haplotypes which are designated by addition of a lowercase letter to the phylogenetic species designation (e.g., FIESC 5-a, FIESC 5-b, FIESC 5-c). FIESC species were resolved into two major clades: Equiseti and Incarnatum. The Equiseti clade was represented by 15 phylogenetic species, only three of which were linked to published species: F. equiseti (FIESC 14), F. lacertarum (FIESC 4), and F. scirpi (FIESC 9) (O'Donnell et al., 2009). The Incarnatum clade also included 15 phylogenetic species. Fusarium equiseti (Corda) Saccardo (1886) is a soilinhabiting saprophyte able of colonizing roots of agriculturally important crops including cereals. The fungus has also been associated with mycotic infection of humans and other animals (O'Donnell et al., 2009, 2012; Short et al., 2011; van Diepeningen et al., 2015). Fusarium scirpi (Lambotte and Fautrey, 1894) is morphologically similar to F. equiseti, and the two species are frequently confused. However, F. scirpi tends to occur in arid and semi-arid regions, particularly in soil (Leslie and Summerell, 2006).

DNA-based phylogenetic analyses have provided significant insight into genetic diversity and phylogenetic relationships among members of this complex (O'Donnell et al., 2009, 2012). However, plant pathogenicity, plant disease epidemiology, and mycotoxin production profiles of members of the FIESC are poorly understood. Mycotoxin production profiles have been used in polyphasic approaches to understand diversity and species boundaries within Fusarium (Moretti et al., 1996; Watanabe et al., 2013). Over the past two decades, genes responsible for biosynthesis of the Fusarium mycotoxin, such as trichothecenes and fumonisins (Brown et al., 2001; Proctor et al., 2003) have been identified. This has provided a tool to predict mycotoxin production potential of field isolates (Jurado et al., 2005, 2006; Ward et al., 2002). In addition, the proteomic approach of Matrix-Assisted Laser Desorption/ Ionization Time-Of-Flight Mass Spectrometry (MALDI-TOF MS) has emerged as a powerful tool for fast and reliable identification of species within several genera of fungi, including Fusarium (Marinach-Patrice et al., 2009; Rodrigues et al., 2011; Ranque et al., 2014; Silva et al., 2015).

Thus, the objective of this study was to characterize a collection of 69 fusaria from cereals that exhibited FIESC-like morphology. This was accomplished using DNA-based phylogenetic, MALDI-TOF and mass spectrometry-based mycotoxin analyses. The phylogenetic analyses indicate that all of the isolates are members of FIESC, and resolved most of them in four previously described phylogenetic species. However, seven isolates were resolved as a novel species lineage designated FIESC 31. The results also indicate that phylogenetic species can exhibit distinct MALDI-TOF MS spectra. Finally, isolates within and among phylogenetic species can vary in their ability to produce trichothecene mycotoxins. These findings provide important insight into the diversity of FIESC isolates that occur on cereals.

\section{Materials and methods}

\subsection{Fungal strain collection}

Sixty-nine isolates morphologically defined as F. equiseti and other FIESC isolated from cereals, mainly maize, oat and wheat, were included in the present study (Table 1). Strains were selected from the Agri-Food

Table 1

List of isolates analyzed in this study. *: Isolates selected for MALDI-TOF MS analysis $\S$ : Isolates selected for LC MS/MS analysis.

\begin{tabular}{|c|c|c|c|}
\hline ITEM $^{*}$ & Host/Substrate & origin & year \\
\hline 190 & barley & Italy & 1978 \\
\hline 10774 & maize & & 2008 \\
\hline 11037 & maize & Italy & 2008 \\
\hline 11080 & maize & Italy & 2010 \\
\hline $13569^{*} \S-13575^{*} \S-13576-13577$ & maize & Netherlands & 2010 \\
\hline \multicolumn{4}{|l|}{$13580^{*} \S-13585-13601^{*}-13607$} \\
\hline $11285-11294^{*} \S-11296^{*} \S-11297^{*} \S$ & oat & Canada & 2006 \\
\hline $11276^{*} \S-11320^{*} \S-11322^{*} \S-11323$ & oat & Canada & 2008 \\
\hline \multicolumn{4}{|l|}{$11345^{*} \S-11348-11354^{*} \S-11359^{*} \S$} \\
\hline \multicolumn{4}{|l|}{$11363^{*} \S-11392-11393-11400^{*} \S$} \\
\hline \multicolumn{4}{|l|}{$11401^{*} \S-11403^{*} \S-11404^{*} \S-11407^{*} \S$} \\
\hline \multicolumn{4}{|l|}{$11410-11414^{*} \S-11419$} \\
\hline $11417-11418 * \S-11420 * \S-11426 * \S-11427$ & oat & Canada & 2009 \\
\hline $3673-3682$ & rice & Italy & 1999 \\
\hline 6808 & rice & Italy & 2004 \\
\hline 10906 & soil & Italy & \\
\hline 192 & wheat & Italy & 1979 \\
\hline $6461-6464$ & wheat & Italy & 2003 \\
\hline 11189 & wheat & Italy & 2008 \\
\hline $10387-10389-10391^{*} \S-10392 \% \S$ & wheat & Italy & 2009 \\
\hline \multicolumn{4}{|l|}{$10393-10395-10416-10433$} \\
\hline \multicolumn{4}{|l|}{$10440-10445-11126$} \\
\hline 13005 & wheat & Mexico & \\
\hline 10669-10671-10673-10674-10675-10786-10787-10788-10789 & wheat & Spain & 2009 \\
\hline
\end{tabular}

* = ITEM, Agri-Food Toxigenic Fungi Culture Collection, http://www.ispa.cnr.it/Collection 
Table 2

List of Fusarium incarnatum-equiseti species complex reference strains. *: strains selected for MALDI-TOF MS analysis §: strains selected for LC MS/MS analysis.

\begin{tabular}{lll}
\hline species & ITEM & source \\
\hline Fusarium equiseti (FIESC 5) & $7633^{*} \S$ & KSU 11460 \\
Fusarium equiseti (FIESC 5) & $15511^{*}$ & KSU 20979 \\
Fusarium sp. (FIESC 25) & $6748^{*} \S$ & FRC R-4237 \\
Fusarium sp. (FIESC 16) & $7547^{*} \S$ & NRRL 20425 \\
Fusarium scirpi (FIESC 9) & $7621^{*} \S$ & KSU 11409 \\
Fusarium scirpi (FIESC 9) & $15556^{*}$ & KSU 20985 \\
Fusarium scirpi (FIESC 9) & & FRC R-06979 \\
Fusarium sp. (FIESC 12) & & NRRL 13405 \\
Fusarium camptoceras & & NRRL 13381 \\
Fusarium sp. (FIESC 15) & & NRRL 31160 \\
Fusarium sp. (FIESC23) & 7155 & NRRL 20695 \\
Fusarium longipes & & \\
\hline
\end{tabular}

Toxigenic Fungi Culture Collection (ITEM, Institute of Sciences of Food Production, Bari, Italy; http://www.ispa.cnr.it/Collection). They were isolated over a span of 30 years and from a wide range of geographical areas (Canada, Italy, Mexico, The Netherlands, Spain, and Turkey). Eleven other strains used as references and/or outgroups were received from the following culture collections: Fusarium Research Center (FRC) at Pennsylvania State University, State College, Pennsylvania; the ARS Culture Collection (NRRL) at the US Department of Agriculture, National Center for Agricultural Utilization Research in Peoria, Illinois); and the collection at Kansas State University, Manhattan, Kansas (Table 2).

\subsection{DNA extraction}

Fungal isolates were grown in Wickerham medium ( $40 \mathrm{~g}$ of glucose, $5 \mathrm{~g}$ of peptone, $3 \mathrm{~g}$ of yeast extract, $3 \mathrm{~g}$ of malt extract per liter) on a rotary shaker at $120 \mathrm{rpm}$ for $48 \mathrm{~h}$ in the dark. Fresh mycelia were collected by vacuum filtration through No. 4 Whatman filter paper (Whatman Biosystems Ltd., Maidstone, UK), then frozen and lyophilized. Total genomic DNA was extracted using the "Wizard ${ }^{\circledR}$ Magnetic DNA Purification System for Food" kit (Promega, USA) according to the manufacturer's protocol, using $10 \mathrm{mg}$ of dried mycelium.

\subsection{Sequence analysis}

Phylogenetic relationships of isolates were investigated by amplifying and sequencing the housekeeping genes calmodulin (CAL1), the second largest subunit of RNA polymerase II (RPB2), translation elongation factor (TEF1), and $\beta$-tubulin (TUB2), as well as the trichothecene biosynthetic genes TRI1, TRI4, TRI5, TRI8 and TRI11. The sequence for Gene $\mathrm{F}$ was also included in this analysis. Gene $\mathrm{F}$ is predicted to encode a $\mathrm{Zn}$ (II)2Cys6 transcription factor and is located within the TRI cluster in members of the FIESC, but not in cluster of other trichothecene-producing fusaria (Proctor et al., 2009). Primers used to amplify and sequence TRI8, TRI11, and Gene F were designed during the course of this study based on previously described sequences for these genes (Proctor et al., 2009), using the following PCR program: denaturation at $96^{\circ} \mathrm{C}$ for $2 \mathrm{~min} ; 35$ cycles of the denaturation at $94^{\circ} \mathrm{C}$ for $30 \mathrm{~s}$, annealing at $55^{\circ} \mathrm{C}$ for $30 \mathrm{~s}$, extension at $68{ }^{\circ} \mathrm{C}$ for $75 \mathrm{~s}$; and final extension at $68{ }^{\circ} \mathrm{C}$ for $10 \mathrm{~min}$. All other primers have been described previously (Table 3 ). Before sequencing, PCR products were purified with the enzymatic mixture EXO/FastAP (Exonuclease I, FastAP thermosensitive alkaline phosphatase, Thermo Scientific, Lithuania, Europe). Sequence reactions were performed using a BigDye Terminator v3.1 Cycle Sequencing Ready Reaction Kit for both strands, after which they were purified by gel filtration through Sephadex G-50 (5\%) (Amersham Pharmacia Biotech, Piscataway, NJ, USA), before they were analyzed on the 3730xl DNA Analyzer (Applied Biosystems, Foster City, CA, USA). DNA sequences were determined by Sequencing Analysis 5.2 software (Applied Biosystems) and aligned by the Clustal W algorithm (Thompson et al., 1994) with MEGA 5.2 software (Tamura et al., 2011). Representative sequences of the four loci were submitted to GenBank; accession numbers are LN901584-LN901599 for CAL1, LN901600-LN901619 for RPB2, LN901566-LN901583 for TEF1, and LN901620-LN901632 for TUB2 (Table 4).

\subsection{Phylogenetic analyses}

Phylogenetic relationships were inferred with MEGA 5.2 software. Bootstrap analysis (Felsenstein, 1985) was conducted to determine the confidence for the internal nodes using a heuristic search with 1000 replicates. The combined data set consisting of partial sequences of the CAL1, RPB2, TEF1, and TUB2 genes (2454 bp) and of the six TRI genes (4542 bp) were analyzed using Maximum Parsimony. Representative sequences for TRI genes have been submitted to Genbank as accession numbers from LN995562-LN995612. Some phylogenetic analyses also included CAL1, RPB2, TEF1 and TUB2 sequences from previously defined FIESC phylogenetic species. The latter sequences were obtained from GenBank.

Table 3

Sequence of oligonucleotide primers used in this study.

\begin{tabular}{|c|c|c|c|c|c|}
\hline Target gene & Primers & $5^{\prime}>3^{\prime}$ nucleotide sequence & $\mathrm{Ta}\left({ }^{\circ} \mathrm{C}\right)$ & exp. size & references \\
\hline \multirow[t]{2}{*}{ Gene F } & 3891 & GCTGTCAYAGYCAGAAGYTACGATG & 54 & 1200 & This study \\
\hline & 3894 & AGAYATGBAGGACARGGCTTAGGGT & & & \\
\hline \multirow[t]{2}{*}{ TRI1 } & 1285 & GCGTCTCAGCTTCATCAAGGCAKCKAMTGAWTCG & 54 & 1200 & Proctor et al. (2009) \\
\hline & 1292 & CTTGACTTSMTTGGCKGCAAAGAARCGACCA & & & \\
\hline \multirow[t]{2}{*}{ TRI4 } & 2576 & CCAATCAGYCAYGCTRTTGGGATACTG & 55 & 1800 & Proctor et al. (2009) \\
\hline & 2578 & ACCCGGATTTCRCCAACATGCT & & & \\
\hline \multirow[t]{2}{*}{ TRI5 } & 1558 & GGCATGGTCGTGTACTCTTGGGTCAAGGT & 55 & 1300 & Proctor et al. (2009) \\
\hline & 1559 & GCCTGMYCAWAGAAYTTGCRGAACTT & & & \\
\hline \multirow[t]{2}{*}{ TRI8 } & 3904 & GACCAGNAYCACSGYCAACAGTTCAG & 55 & 1200 & This study \\
\hline & 3906 & GAACAGCCRCTCCRWAACTATTGTC & & & \\
\hline \multirow[t]{2}{*}{ TRI11 } & 3895 & TWCCCCACAAGRAACAYCTYGARCT & 54 & 1300 & This study \\
\hline & 3897 & TCCCASACTGTYCTSGCMAGCATCAT & & & \\
\hline \multirow[t]{2}{*}{ CAL1 } & CL1 & GARTWCAAGGAGGCCTTCTC & 55 & 650 & O'Donnell et al. (2000) \\
\hline & CL2 & TTTTGCATCATGAGTTGGAC & & & \\
\hline \multirow[t]{2}{*}{ RPB2 } & $5 \mathrm{~F}$ & GAYGAYMGVGATCAYTT & 57 & 1000 & Liu et al. (1999) \\
\hline & 7cR & CCCATRGCTTGYTTRCCCAT & & & \\
\hline \multirow[t]{2}{*}{ TEF1 } & $\mathrm{EF} 1$ & ATGGGTAAGGARGACAAGAC & 54 & 700 & O'Donnell et al. (1998) \\
\hline & $\mathrm{EF} 2$ & GGARGTACCAGTSATCATGTT & & & \\
\hline \multirow[t]{2}{*}{ TUB2 } & $\mathrm{Bt} 2 \mathrm{a}$ & GGTAACCAAATCGGTGCT & 60 & 400 & Glass and Donaldson (1995) \\
\hline & $\mathrm{Bt} 2 \mathrm{~b}$ & ACCCTCAGTGTAGTGACCCTYTGGC & & & \\
\hline
\end{tabular}


Table 4

List of GenBank accession numbers provided for CAL1, RPB2, TEF1, and TUB2.

\begin{tabular}{|c|c|c|c|c|}
\hline ITEM & CAL1 & TEF1 & $R P B 2$ & TUB2 \\
\hline 6464 & LN901584 & & LN901600 & \\
\hline 6748 & LN901598 & LN901582 & LN901618 & LN901631 \\
\hline 6808 & LN901591 & LN901575 & & \\
\hline 7155 & LN901597 & LN901581 & LN901617 & LN901630 \\
\hline 7547 & LN901596 & LN901580 & LN901616 & LN901629 \\
\hline 7621 & LN901599 & LN901583 & LN901619 & LN901632 \\
\hline 7633 & LN901595 & LN901579 & LN901615 & LN901628 \\
\hline 10387 & LN901585 & & & \\
\hline 10392 & LN901592 & LN901576 & LN901610 & LN901625 \\
\hline 10393 & LN901586 & LN901566 & LN901601 & \\
\hline 10395 & LN901593 & LN901577 & & \\
\hline 10416 & & LN901567 & & \\
\hline 10433 & & & LN901602 & \\
\hline 10445 & & LN901568 & LN901603 & \\
\hline 10674 & & LN901569 & & \\
\hline 10675 & LN901590 & LN901573 & LN901607 & LN901623 \\
\hline 10787 & & & & LN901620 \\
\hline 10789 & LN901587 & & LN901604 & LN901621 \\
\hline 11294 & & LN901571 & & \\
\hline 11296 & LN901589 & LN901572 & LN901606 & LN901622 \\
\hline 11320 & & & LN901608 & \\
\hline 11363 & & LN901574 & LN901609 & \\
\hline 11392 & & & LN901605 & \\
\hline 11401 & LN901594 & LN901578 & LN901611 & LN901626 \\
\hline 13005 & LN901588 & LN901570 & & \\
\hline 13569 & & & LN901612 & \\
\hline 13580 & & & LN901613 & LN901627 \\
\hline 13585 & & & & LN901624 \\
\hline 13601 & & & LN901614 & \\
\hline
\end{tabular}

\subsection{MALDI-TOF MS analysis}

A subset of 25 of the 69 isolates was selected for this investigation (Table 1) based on a phylogenetic assessment. Six strains, ITEM 7633 and 15511 (F. equiseti), ITEM 7621 and 15556 (F. scirpi), ITEM 7547 (in other papers named as F. incarnatum, O'Donnell et al., 2007) and 6748 (in other papers named as F. semitectum, Susca et al., 2015) were used as species-reference strains. Seven-day-old spores of each isolate suspended in $0.5 \mathrm{~mL}$ of $0.2 \%$ agar were used for a single point inoculum which were incubated at $25^{\circ} \mathrm{C}$ on 6 -cm diameter Petri dishes with malt extract agar ( $20 \mathrm{~g}$ of malt, $20 \mathrm{~g}$ of glucose, $1 \mathrm{~g}$ peptone, $20 \mathrm{~g}$ of agar). Approximately $1 \mu \mathrm{g}$ of mycelium and spores (from colonies not older than $72 \mathrm{~h}$ ) of each fungus was directly transferred from the culture plate to the MALDI flex target plate. Matrix solution $(0.5 \mu \mathrm{L}$ of $75 \mathrm{mg} / \mathrm{mL} 2.5$ dihydroxybenzoic acid in ethanol/water/acetonitrile [1:1:1]) was immediately added and mixed gently.

Escherichia coli strain DH5 $\alpha$ was used as a control for in loco protein extraction and MALDI-TOF MS calibration. Cells were grown and maintained on Luria-Bertani (LB) agar medium (LB: $10 \mathrm{~g}$ of bacto-tryptone, $5 \mathrm{~g}$ of bacto-yeast extract, $10 \mathrm{~g}$ of $\mathrm{NaCl}, 15 \mathrm{~g}$ bacto-agar per liter). $E$. coli $\mathrm{DH} 5 \alpha$ incubation was standardized at $20 \mathrm{~h}$ and grown aerobically at $37{ }^{\circ} \mathrm{C}$. All cultures were checked for purity prior to use and were subcultured at least once prior to analysis. Approximately $1 \mu \mathrm{g}$ of cells (about $10^{7}$ cells per sample) was directly transferred from a single colony and spotted onto the 48 well MALDI flex target plate. The matrix solution was added and samples were air dried at room temperature. Each sample was spotted in duplicate to test reproducibility.

During the analyses all solutions were freshly prepared and kept at $5{ }^{\circ} \mathrm{C}$. The analyses were performed on a Shimadzu Axima LNR system (Kratos Analytical, Manchester, U.K.) equipped with a nitrogen laser (337 nm), where the laser intensity was set just above the threshold for ion production. Ions of 12 well-defined ribosomal proteins of intact E. coli DH5 $\alpha$ cells $(4365.4,5096.8,5381.4,6241.4,6255.4,6316.2$, 6411.6, 6856.1, 7158.8, 7274.5, 7872.1, 9742 and 12,227.3 Da) were used as external calibrants. The mass spectra based on the mass range from 2 to $20 \mathrm{kDa}$ were recorded using the linear mode with a delay of
$104 \mathrm{~ns}$ and an acceleration voltage of $+20 \mathrm{kV}$. The final spectra were generated by summing 20 laser shots accumulated per profile and 50 profiles produced per sample, which led to 1000 laser shots per summed spectrum.

Resulting ion peak lists were exported to the Spectral Archive And Microbial Identification System (SARAMISTM ${ }^{\mathrm{TM}}$, AnagnosTec GmbH, Potsdam, Germany) software package which enabled final microbial identification. In SARAMISTM, peak lists of individual samples were compared to a database generating a ranked list of matching spectra. For each sample analyzed, only those data with match $\geq 99 \%$ spectral similarity were used. This software uses a point system based on ion peak lists with mass signals weighted according to their specificity. The similarity between individual spectra is expressed as the relative or absolute number of matching mass signals after subjecting the data to a single link agglomerative clustering algorithm. Microbial identifications by the SARAMIS ${ }^{\mathrm{TM}}$ package are based on the presence or absence of each ion peak in the spectra. A dendrogram of spectral proximity between isolates was created with this software (Santos et al., 2015).

\subsection{LC-(TOF-)MS(/MS) analysis}

Based on the phylogenetic assessment, a subset of 28 isolates (Tables 1 and 2) was selected for multitoxin analysis through a combination of untargeted LC-TOF-MS(/MS) for screening and targeted LC-MS/MS for confirmation.

\subsection{Untargeted analysis of mycotoxins by LC-TOF-MS(/MS)}

LC-Q-TOF (SCIEX, Framingham, MA, USA) was used for screening metabolites produced by the isolates. Chromatographic separation was achieved on a Zorbax Eclipse Plus C18 column $(100 \mathrm{~mm} \times 2.1 \mathrm{~mm}, 1.8 \mu \mathrm{m})$ (Agilent Technologies, Santa Clara, CA, US) at $40{ }^{\circ} \mathrm{C}$, with a flow rate of $0.4 \mathrm{~mL} / \mathrm{min}$. The mobile phase consisted of $(\mathrm{A})$ water/methanol $(\mathrm{v} / \mathrm{v}, 5 / 95)$ containing $0.1 \%$ formic acid and $10 \mathrm{mM}$ ammonium formate and (B) water/methanol $(v / v, 95 / 5)$ containing $0.1 \%$ formic acid and $10 \mathrm{mM}$ ammonium formate. A linear gradient elution program was applied as follows: 0 min $0 \%$ B, 0.5 min $0 \%$ B, 7 min 99\% B, 9 min 99\% B, 10 min 0\% B and hold on for a further 4 min for re-equilibration, giving a total run time of $14 \mathrm{~min}$. The injection volume was $5 \mu \mathrm{L}$. Triple TOF 4600 was operated in positive electrospray ionization mode $(\mathrm{ESI}+)$ for MS analysis with scan range of 50-1600 $(\mathrm{m} / \mathrm{z})$. The parameters were as follows. Source type: DuoSpray ion source, source temperature: $500{ }^{\circ} \mathrm{C}$, injection manifold: bypass. The major primary ions (with responses $>10.000$ ) were further investigated by $\mathrm{MS}^{2}$ for product ions with following parameters: collision energy (35 eV), cycle number (1637), period cycle time (470 m9 s), pulser frequency $(12.576 \mathrm{kHz})$, accumulation time $(50 \mathrm{~ms})$. Calculation of the accurate mass of the analytes and their product ions were performed by PeakView software (version 1.2, SCIEX, Framingham, MA, USA). The accurate mass of the analyte was calculated by the precursor ions of $[\mathrm{M}+\mathrm{H}]^{+}$and by $[\mathrm{M}+\mathrm{Na}]^{+},[\mathrm{M}-\mathrm{OH}]^{+},[\mathrm{M}+\mathrm{NH} 4]^{+}$, and the precursor ions of their isotopical patterns with peak spacing tolerance of $5.0 \mathrm{ppm}$. The compounds were identified by comparing them with the library established in our lab.

\subsection{Targeted LC-MS/MS confirmatory analysis}

Reference DON, 15-ADON, 3-ADON, chaetoglobosin A (CHA), diacetoxyscirpenol (DAS), DON-3-glucoside (DON-3G), fusarenone $\mathrm{X}$ (FUS-X), mycophenolic acid (MPA), neosolaniol (NEO), and nivalenol (NIV) standards were purchased from Sigma-Aldrich (St. Louis, MO, USA). Ultra-high performance liquid chromatography (UHPLC) analysis was performed with a Waters Acquity UHPLC system (Waters, Zellik, Belgium). Analytes were separated on an Acquity CSH Fluoro-Phenyl column (150 mm*2.1 mm, $1.7 \mu \mathrm{m})$ (Waters, Zellik, Belgium) maintained at $40{ }^{\circ} \mathrm{C}$, with a mobile phase flow rate of $0.40 \mathrm{~mL} / \mathrm{min}$. The 


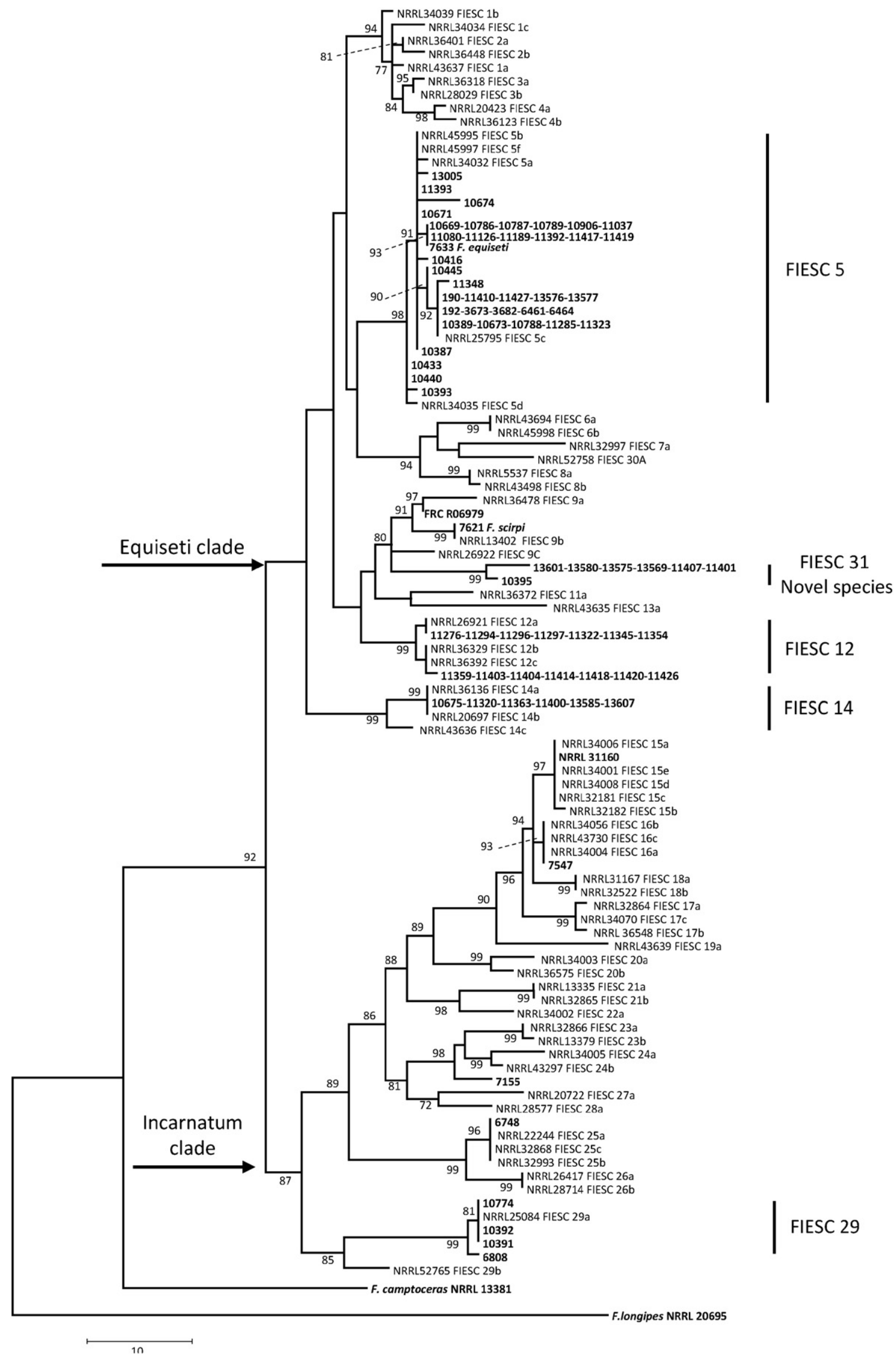

Fig. 1. Phylogenetic tree inferred from partial TEF1 sequences from members of the Fusarium incarnatum-equiseti species complex isolates analyzed in this study. Sequences generated in the present study are in bold, the other sequences were downloaded from Fusarium-ID. The evolutionary history was inferred by using the Maximum Parsimony method. Numbers on branches are bootstrap values based on 500 pseudoreplicates. 
mobile phase consisted of (A) water containing $5 \mathrm{mM}$ ammonium acetate and $0.3 \%$ acetic acid and (B) methanol containing $5 \mathrm{mM}$ ammonium acetate and $0.3 \%$ acetic acid. A linear gradient elution program was applied as follows: initial 10\% B, 2 min 10\% B, 5 min 33\% B, 5.1 min $75 \%$ B, 7 min 75\% B, 8 min 99\% B, 9 min 99\% B, 11 min 10\% B, and held for a further $3 \mathrm{~min}$ for re-equilibration, giving a total run time of $14 \mathrm{~min}$. The injection volume was $10 \mu \mathrm{L}$. The MS/MS determination was performed on a Quattro Premier XE tandem quadrupole mass spectrometer (Waters, Milford, MA, USA) equipped with an ESI + source. The parameters used for ionization were as follows: capillary voltage ( $3.5 \mathrm{kV})$, source temperature $\left(120^{\circ} \mathrm{C}\right)$, and desolvation temperature $\left(350^{\circ} \mathrm{C}\right)$. The cone and desolvation gas flow were 50 and $800 \mathrm{~L} / \mathrm{h}$, respectively. Analyte confirmation was performed in multiple reaction monitoring (MRM) mode using two product ions. The optimum conditions were set for each mycotoxin by direct infusion. Data acquisition and processing were performed using MassLynx 4.0 (Waters ).

The 28 isolates were grown on PDA for 1 and 2 weeks and then transferred from the plates into $50 \mathrm{~mL}$ extraction tubes. The extraction solvent $(20 \mathrm{~mL})$, consisted of water/acetonitrile $(84 / 16, v / \mathrm{v})$ was added to each of the tubes. The mixture was shaken for $60 \mathrm{~min}$, and the supernatant was transferred to a new extraction tube and centrifuged at $5000 \mathrm{~g}$ for $5 \mathrm{~min}$. A aliquot $(5 \mathrm{~mL})$ of the supernatant was collected and dried under nitrogen gas at $40{ }^{\circ} \mathrm{C}$. The residues were then reconstituted using different solutions for LC-TOF-MS and LC-MS/MS analysis, respectively. For LC-TOF-MS, $0.2 \mathrm{~mL}$ of water/acetonitrile/ methanol $(40 / 30 / 30)$ solution was used to dissolve the residues, while for LC-MS/MS, $1 \mathrm{~mL}$ of the water solvent (A) containing $5 \mathrm{mM}$ ammonium acetate and $0.3 \%$ acetic acid and solvent (B) methanol containing $5 \mathrm{mM}$ ammonium acetate and $0.3 \%$ acetic acid $(70 / 30, v / \mathrm{v})$ were used. After vortexing for 2 min followed by centrifugation at $3200 \mathrm{~g}$ for $5 \mathrm{~min}$, the reconstituted extracts were filtered $(0.22 \mu \mathrm{m}$, nylon filter $)$ (Millipore, Billerica, MA, USA) and then centrifuged for $10 \mathrm{~min}$ at $10,000 \mathrm{~g}$. The filtrate was collected in LC-MS vials for analysis.

\section{Results}

\subsection{Identification of cereal isolates as members of the FIESC}

Initial assessment of 69 isolates of Fusarium recovered from cereals grown in Canada, Italy, Mexico, The Netherlands, Spain and Turkey (Table 1 ) indicated that they were morphologically similar to F. equiseti and related species within the FIESC. To determine whether these isolates were members of FIESC, we conducted a BLASTn analysis of the TEF1 sequence from each isolate against the Fusarium-ID database (Geiser et al., 2004). The analysis indicated that the TEF1 sequences were most similar to members of the FIESC.

To further resolve the identity of the 69 isolates, we conducted a phylogenetic analysis with TEF1 sequences from the isolates as well as from previously defined phylogenetic species within the FIESC (O'Donnell et al., 2009, 2012). This analysis indicated 65 isolates were nested within the Equiseti clade and only four isolates grouped with the Incarnatum clade (Fig. 1). The evolutionary history was inferred using the Maximum Parsimony (MP) and Maximum Likelihood methods (data not shown) and the bootstrap support from analysis of the individual genes and combined gene sequences are shown in Table 5. MP Tree \#1 out of 74 most-parsimonious trees (length = 427 ) is shown (Fig. 1). The consistency index is (0.58), the retention index is (0.91), and the composite index is $0.57(0.53)$ for all sites and parsimony-informative sites (in parentheses). For the isolates resolved within the Equiseti clade, 58 were further resolved into three previously defined unnamed phylogenetic species: FIESC 5, FIESC 12 and FIESC 14 (Fig. 1). The four isolates resolved within the Incarnatum clade were identified as phylogenetic species FIESC 29 (Fig. 1). The majority of the isolates examined here were identified as FIESC 5. Most of these isolates were recovered from wheat in Italy, but some were recovered from other hosts and/or geographic origins. The 14 FIESC 12 isolates were all recovered from oats in Canada. Isolates identified as FIESC 14 ( $n=$ 6 ) were recovered from multiple hosts and locations: two isolates were from maize in The Netherlands, three were from oats in Canada, and one was from wheat in Spain. The four FIESC 29 isolates also had multiple origins: one was from rice and two were from wheat in Italy, and one was from maize in Turkey.

In the TEF1 phylogeny, seven isolates (10395, 11401, 11407, 13569, 14575,13580 and 13601) nested within the Equiseti clade appeared to represent a novel phylogenetic species that was strongly supported by bootstrapping (bootstrap value 100). In order to better assess the species status of this putatively novel lineage, we conducted phylogenetic analyses of portions of CAL1 and RPB2 (Supplementary Fig. S1; Supplementary Fig. S2) and a 4-gene combined data set (Fig. 2). Maximum Parsimony tree \# 1 out of 21 most-parsimonious trees (length $=851$ ) is shown (Fig. 1). The consistency index is (0.54), the retention index is (0.89), and the composite index is $0.63(0.48)$ for all sites and

Table 5

Summary sequence data and tree statistics for the three loci and the combined data set obtained with Maximum Likelihood and Maximum Parsimony.

\begin{tabular}{|c|c|c|c|c|c|c|c|c|}
\hline Gene & size & No. of PICs ${ }^{a}$ & Consistency index & Retention index & MPT length ${ }^{b}$ & Phylogenetic species & $\mathrm{ML}^{\mathrm{c}}$ & $\mathrm{MP}^{\mathrm{d}}$ \\
\hline \multirow[t]{5}{*}{ TEF1a } & 601 & 79 & 0.58 & 0.91 & 851 & FIESC 5 & 97 & 98 \\
\hline & & & & & & FIESC 12 & 99 & 99 \\
\hline & & & & & & FIESC 14 & 100 & 99 \\
\hline & & & & & & FIESC 29 & 100 & 99 \\
\hline & & & & & & Novel/FIESC 31 & 100 & 99 \\
\hline \multirow[t]{5}{*}{ CAL1 } & 652 & 59 & 0.58 & 0.94 & 335 & FIESC 5 & 83 & 70 \\
\hline & & & & & & FIESC 12 & 90 & 82 \\
\hline & & & & & & FIESC 14 & 93 & $<70$ \\
\hline & & & & & & FIESC 29 & 95 & 97 \\
\hline & & & & & & Novel/FIESC 31 & 99 & 98 \\
\hline \multirow[t]{5}{*}{ RPB2 } & 790 & 55 & 0.56 & 0.93 & 286 & FIESC 5 & $90 / 99$ & $90 / 98$ \\
\hline & & & & & & FIESC 12 & 99 & 98 \\
\hline & & & & & & FIESC 14 & 81 & 78 \\
\hline & & & & & & FIESC 29 & 99 & 100 \\
\hline & & & & & & Novel/ FIESC 31 & 71 & 78 \\
\hline \multirow{5}{*}{$\begin{array}{l}\text { Combined } \\
\text { data set }\end{array}$} & 2454 & 255 & 0.53 & 0.89 & 427 & FIESC 5 & 74 & 99 \\
\hline & & & & & & FIESC 12 & 100 & 100 \\
\hline & & & & & & FIESC 14 & 100 & 100 \\
\hline & & & & & & FIESC 29 & 100 & 100 \\
\hline & & & & & & Novel/ FIESC 31 & 100 & 100 \\
\hline
\end{tabular}

\footnotetext{
number of Parsimony Informative Characters (PIC).

b length (number of steps) of most parsimonious tree.

c maximum likelihood bootstrap value.

d maximum parsimony bootstrap value.
} 


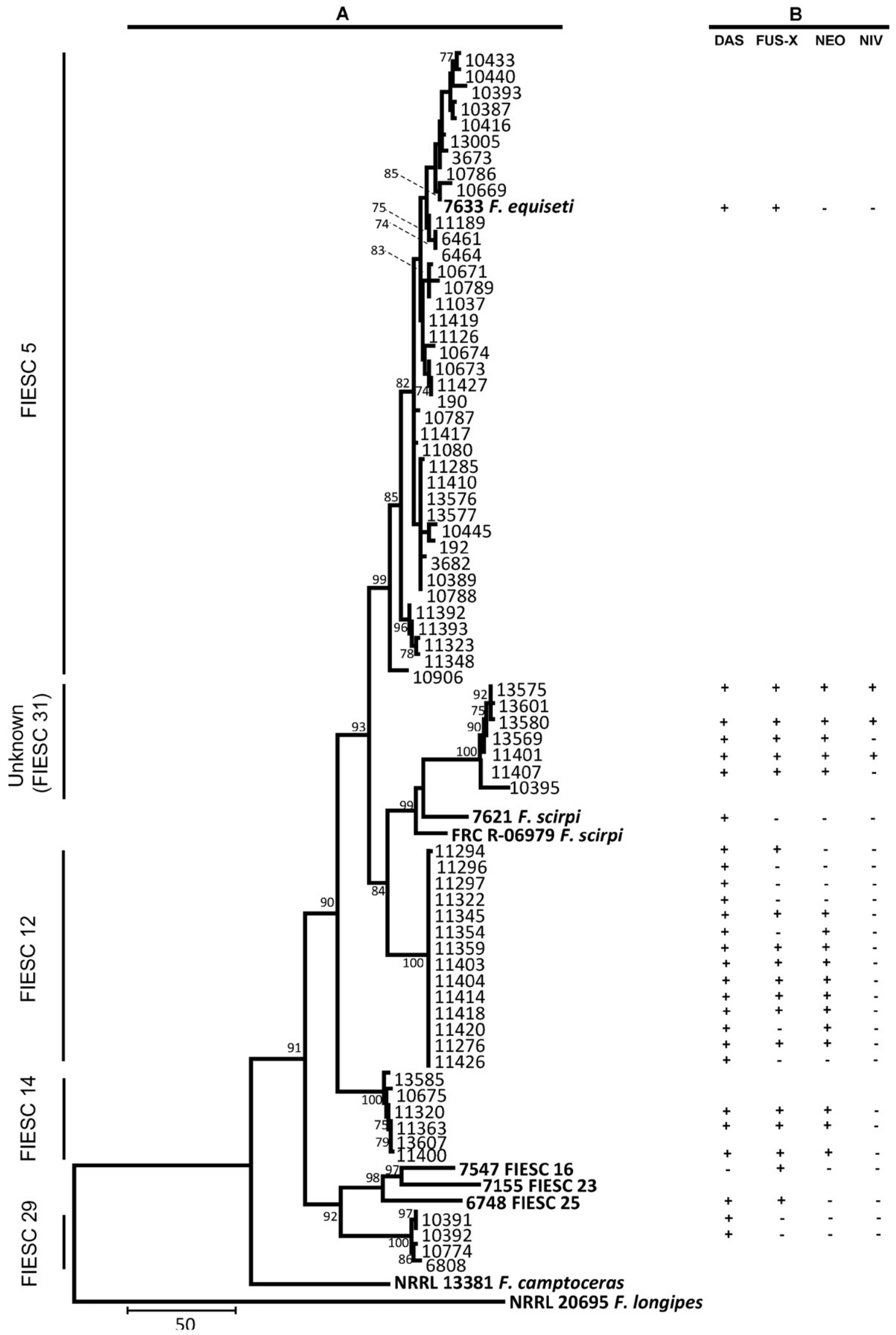

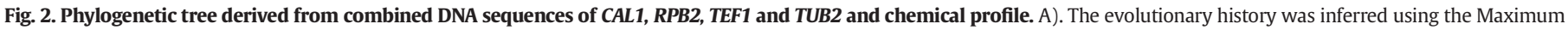

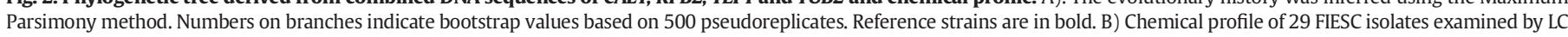
MS/MS. 
parsimony-informative sites (in parentheses). TUB2 sequence data were not available for all reference FIESC strains, so a tree showing the relationship of TUB2 sequences from the cereal isolates to those of reference FIESC strains is not presented. Bootstrap analysis of CAL1 and RPB2 and of the four-gene dataset also supported grouping of cereal isolates into FIESC 5, FIESC 12, FIESC 14 or FIESC 29 (81-100\% bootstrap) (Supplemental Figs. S1 and S2). Bootstrap analysis of CAL1 (98\% bootstrap), RPB2 (78\% bootstrap), and the combined 4-gene dataset (100\% bootstrap) support the genealogical exclusivity of the novel lineage, which we designate as phylogenetic species FIESC 31. Although analysis of the combined CAL1, RPB2, TEF1 and TUB2 dataset did not include reference sequences for FIESC 5, FIESC 12, FIESC 14 and FIESC 29, bootstrap analysis of the combined dataset provided high levels of support (100\% bootstrap) for grouping the cereal isolates in to distinct clades (Fig. 2), which almost certainly correspond to FIESC 5, FIESC 12, FIESC 14 and FIESC 29 based on comparison of the four-gene tree (Fig. 2) to singlegene trees that included reference sequences (Figs. S1 and S2).

The analysis of intra-species variability for FIESC 5 (39 isolates) was overlooked, selecting only 1 strain (species reference ITEM 7633 with formal name F. equiseti), as representative of the group, widely analyzed by Marın et al., 2012; Kosiak et al., 2005; Jurado et al., 2006. Further analysis with different approaches were conducted on remnant 30 isolates, with slight variations in the number for each different approach.

\subsection{MALDI-TOF MS analysis}

Fig. 4 shows the dendrogram resulting from MALDI-TOF MS analysis of 25 selected isolates from our collection of cereal isolates as well as reference strains of six species: F. equiseti ITEM 7633 and ITEM 15511, F. scirpi ITEM 7621 and ITEM 15556, ITEM 7547, and ITEM 6748. In the dendrogram, the similarity between individual spectra is expressed as the relative or absolute number of matching mass signals, based on the presence or absence of each ion peak in the spectra. Each spectrum is built summing all ion peaks that appeared in 1000 shots, and differences between replicates are due to peaks with low intensity that did not appear in all replicates.
The dendrogram showed low percentage of mass similarity for some replicates (ITEM 6748, ITEM 15511, and ITEM 10392), but this low number of similar peaks was enough to differentiate the strains. In this analysis, the following distribution for the field isolates was obtained: FIESC 25 (ITEM 6748) and FIESC 16 (ITEM 7547) show clear separation based on the proteomic profile. Moreover, good clustering of strains was obtained for strains from the same haplotype, all isolates within the novel species, FIESC 31, and most isolates within FIESC 12. For the other haplotypes, only two or three isolates were tested. Except within FIESC 14, all isolates within the same haplotype clustered together. If different strains from a certain species produce different metabolites (with different intracellular proteins), it will reduce the similarity between different strains, resulting in the clustering of strains of the same phylogenetic species into different clades. Even so, it was possible to clearly differentiate FIESC 31, corroborating data obtained by other means.

\subsection{LC-(TOF-) MS(/MS) analysis}

Untargeted LC-TOF-MS(/MS) analysis originally revealed the possible presence of eight mycotoxins (15-ADON, 3-ADON, CHA, DAS, FUS$\mathrm{X}, \mathrm{MPA}, \mathrm{NEO}$, and NIV), however, targeted LC-MS/MS with reference standards could not confirm all of them. The chemical profile of 28 of the FIESC isolates is shown in Fig. 2. DON, 3-ADON, 15-ADON, DON3G, CHA, and MPA were absent in all of the isolates analyzed. DAS was produced by all of the isolates, except the FIESC 16 (ITEM 7547). NEO was detected in all of the FIESC 31 and FIESC 14 isolates, in 9 of 14 FIESC 12 isolates, while it was not detected in F. scirpi reference strain ITEM 7621, F. equiseti reference strain ITEM 7633, and reference strains ITEM 6748 and ITEM 7547, or FIESC 29 isolates (ITEM 10391 and ITEM 10392). FUS-X was produced by 19 of the 28 isolates tested. NIV was produced by only 3 isolates, all of which were FIESC 31 (ITEM 11401, 13575 and 13580). Eighteen isolates produced both type A (NEO/DAS) and type $B(\mathrm{NIV} / \mathrm{FUS}-\mathrm{X})$ trichothecenes, ITEM 7547 produced only type B (FUS-X) trichothecenes and 9 isolates produced only type A (DAS/NEO) trichothecenes.

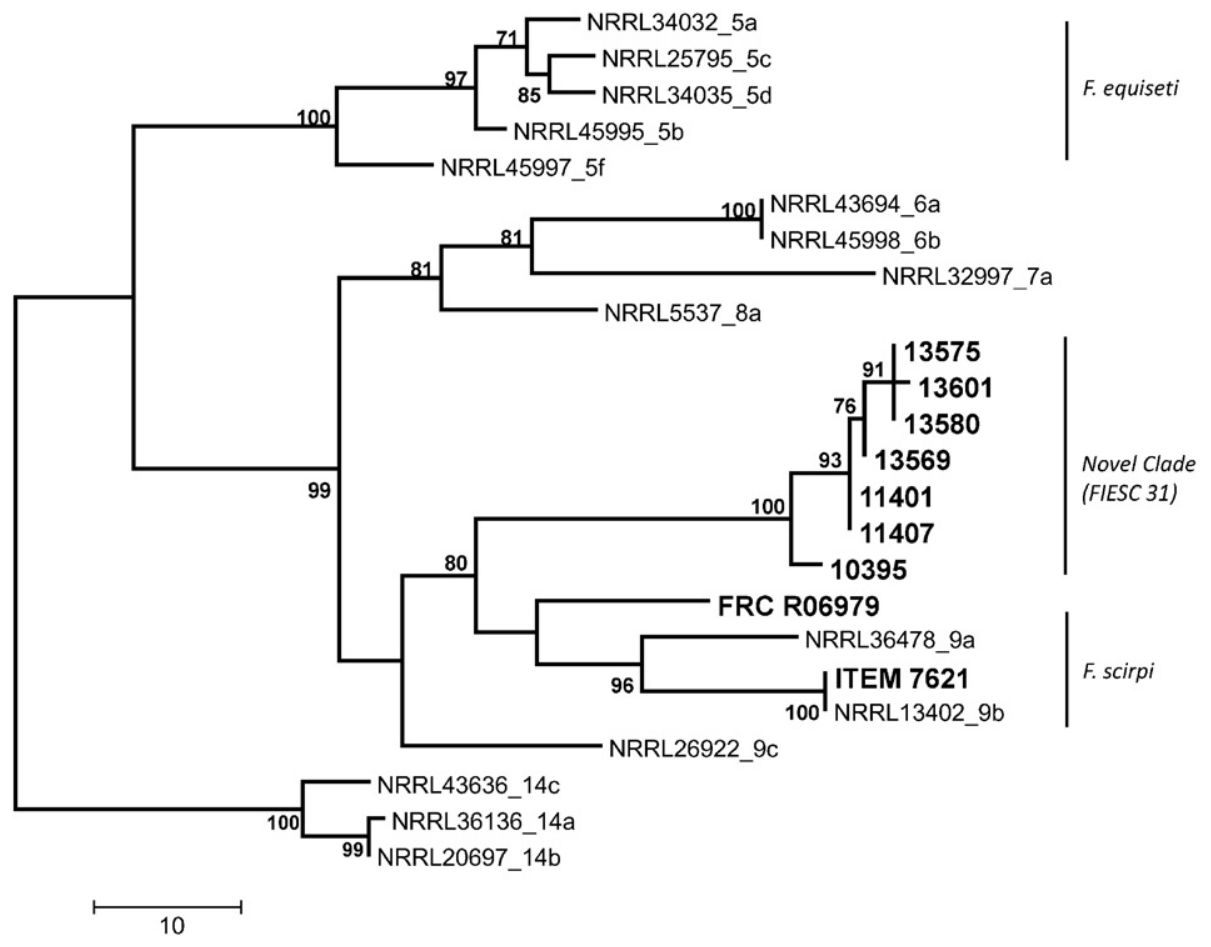

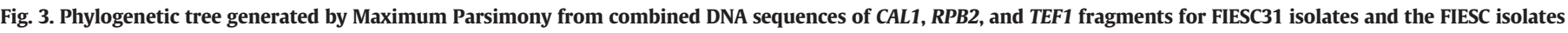

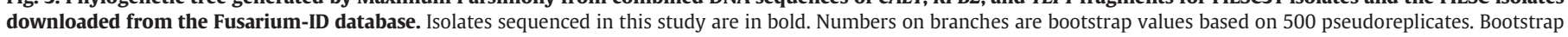
values $\geq 70 \%$ were considered significant. Consistency index $(\mathrm{CI})=0.77$, retention index $(\mathrm{RI})=0.92$. 


\subsection{Analysis of trichothecene biosynthetic genes}

Because isolates from cereals produced trichothecenes, we analyzed variation in select TRI genes in a subset of isolates from our collection. The subset consisted of 26 isolates that represented the range of phylogenetic diversity within the collection. We amplified and sequenced positions of TRI1, TRI4, TRI5, TRI8 and TRI11, as well as the putative TRI Gene F. Six strains did not yield amplicons for one or more of the targeted TRI genes (Supplementary Table S1). As a result, these strains were excluded from phylogenetic analysis of the combined TRI-gene dataset (Fig. 5). Trees generated from individual TRI genes using Maximum Parsimony analysis were also provided as supplementary material (Supplementary Figs. S3-S8).

The phylogenetic tree inferred using Maximum Parsimony analysis of the combined data set of the selected TRI genes was concordant with the species phylogeny derived from housekeeping gene sequences (Fig. 5). For example, the TRI phylogeny resolved the Equiseti and Incarnatum clades. In addition, the TRI phylogeny resolved isolates from different phylogenetic species (FIESC 5, FIESC 12, FIESC 14, FIESC 29 and FIESC 31) into separate and well-supported clades. Finally, the TRI phylogeny resolved FIESC 12 and FIESC 31 isolates as well as a FIESC 9/F. scirpi reference strain into a clade independent of the FIESC 5 and FIESC 14 isolates (Fig. 5, Supplementary Figs. S3-S8).

\section{Discussion}

In the present study, we used for the first time a polyphasic approach to characterize a collection of FIESC isolates recovered from cereals grown in Western Europe, North America and Turkey. The primary focus of this research was to expand our knowledge of FIESC fungi occurring on crops, and in particular on worldwide cereals. Published studies on this group used only a single approach, focusing on phylogenetics relationships and species identification (O'Donnell et al., 2009, 2012; Castellá and Cabañes, 2014) and overlooking mycotoxin production, or alternatively focused on mycotoxin production and overlooked ascertaining phylogenetic species identity (Marın et al., 2012; Kosiak et al., 2005).

Within the FIESC, DNA-based phylogenetic analysis resolved most of the isolates into four previously described phylogenetic species. Over half of the isolates (55\%) were from the same species, FIESC 5. Our study identified a group of seven isolates that constitute a novel species lineage within FIESC. In phylogenetic analyses with the four housekeeping genes, either separately or in combination, these seven isolates were resolved as a well-supported clade that was distinct from all previously reported species within the FIESC (Figs. 1-3, Supplementary Fig. S1; Supplementary Fig. S2). The seven isolates were also resolved as an exclusive and well-supported clade in phylogenetic analysis of TRI genes (Fig. 5, Supplementary Figs. S3-S8), and as a distinct clade in the MALDI-TOF analysis (Fig. 4). Furthermore, three of the seven isolates exhibited a trichothecene production profile (chemotype) that was distinct from all other isolates examined in that they produced NIV in addition to DAS, NEO and FUS-X (Fig. 2). Based on these data, we propose that the seven isolates constitute a novel phylogenetic species of FIESC, which we designate as FIESC 31.

Data obtained from MLST, MALDI-TOF MS, and untargeted LC-TOFMS(/MS) and targeted LC-MS/MS analysis provided tools for better defining biological and toxicological significance of species and haplotypes of FIESC. Moreover, species-specific patterns of proteins in the MALDITOF analysis confirmed the identification of the phylogenetic species, thereby providing another method to distinguish between members of FIESC and that can be used in combination with MLST data. On the other hand, the mycotoxin production profiles were variable among isolates, and were not consistent within haplotypes, phylogenetic species or groups of closely related species (Fig. 2).

To date, studies on genetic diversity within the FIESC have included single nuclear gene sequences (Kristensen et al., 2005; Marın et al.,
2012; Castellá and Cabañes, 2014) or multilocus sequencing approaches (O'Donnell et al., 2009). Our polyphasic approach was applied to isolates from agricultural sources, naturally occurring in fields. The MLST analysis was not only a valuable tool for identifying a novel phylogenetic species within the complex, but also allowed us to assign isolates to four phylogenetic species (FIESC 5, FIESC 12, FIESC 14, and FIESC 29) previously described by O'Donnell et al. (2009, 2012). It is noteworthy that $94 \%$ of the cereal isolates examined here were members of the Equiseti clade; whereas only $6 \%$ were members of the Incarnatum clade. Kosiak et al. (2005) described two distinct populations of $F$. equiseti, Type 1 and Type 2, isolated from Norwegian cereals. These isolates were later identified by Castellá and Cabañes (2014) as FIESC 14 and FIESC 5, respectively. In our study, the most frequently occurring species was FIESC 5, which is in agreement with an analysis of FIESC isolates from Spanish wheat (Castellá and Cabañes, 2014) and an analysis of FIESC isolates from Sardinian soil (Balmas et al., 2010). Thus, three studies indicate that FIESC 5 is a dominant species associated with cereals or soil from cereal fields.

To our knowledge, the current study represents the first report of the occurrence of FIESC 5, FIESC 12, and FIESC 14 in Canada. In addition, as far as we are aware, this study is the first report of the occurrence of FIESC 12 on cereals; previously it was reported only from human tissues in Germany (O'Donnell et al., 2009). The four FIESC 29 isolates originated from regions with warmer climates (Italy and Mexico), similar to those isolated from wheat reported by Castellá and Cabañes' (2014). It remains to be determined whether FIESC 29 occurs more frequently in warmer than in cooler climates.

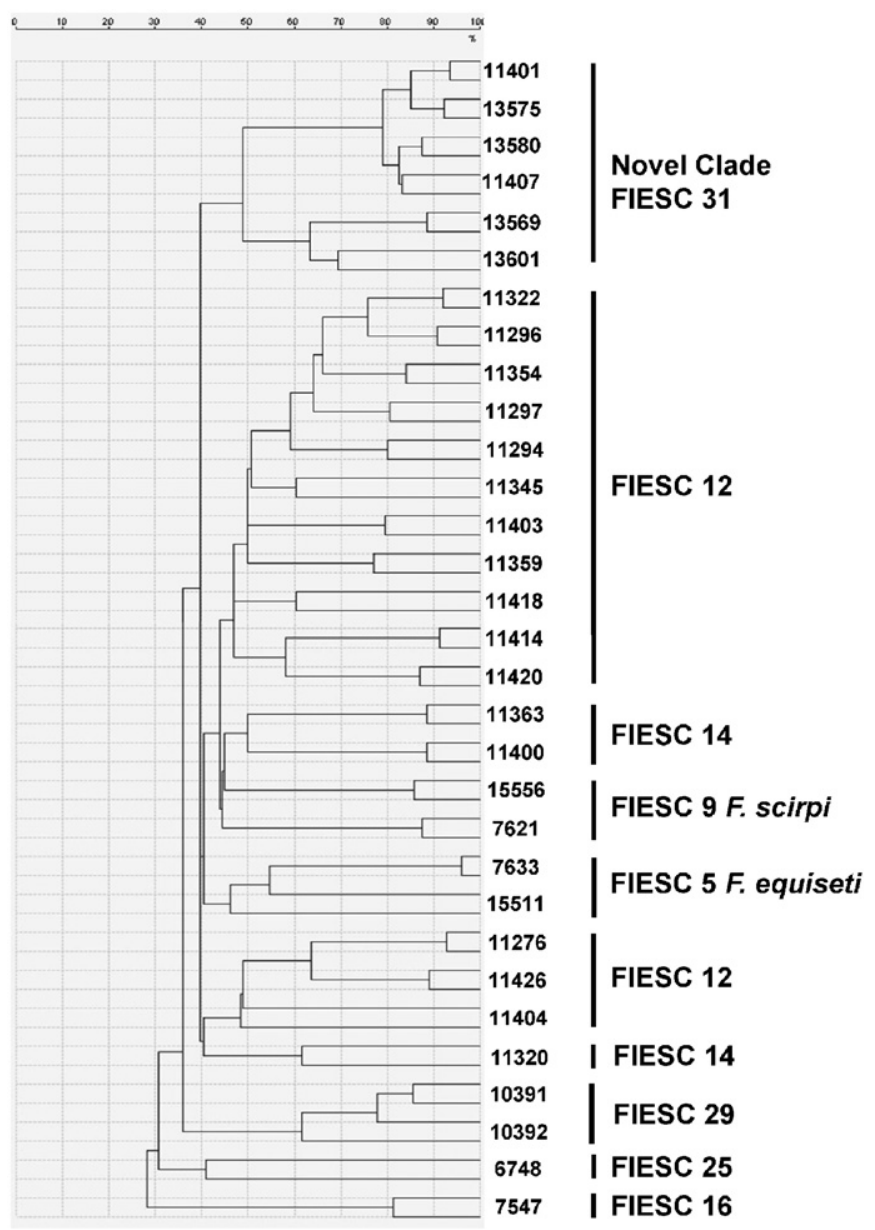

Fig. 4. Dendrogram based on single-linkage cluster analysis of mass spectra of 25 FIESC isolates obtained by MALDI-TOF MS analysis. Distances were measured as percentage of mass similarity. 


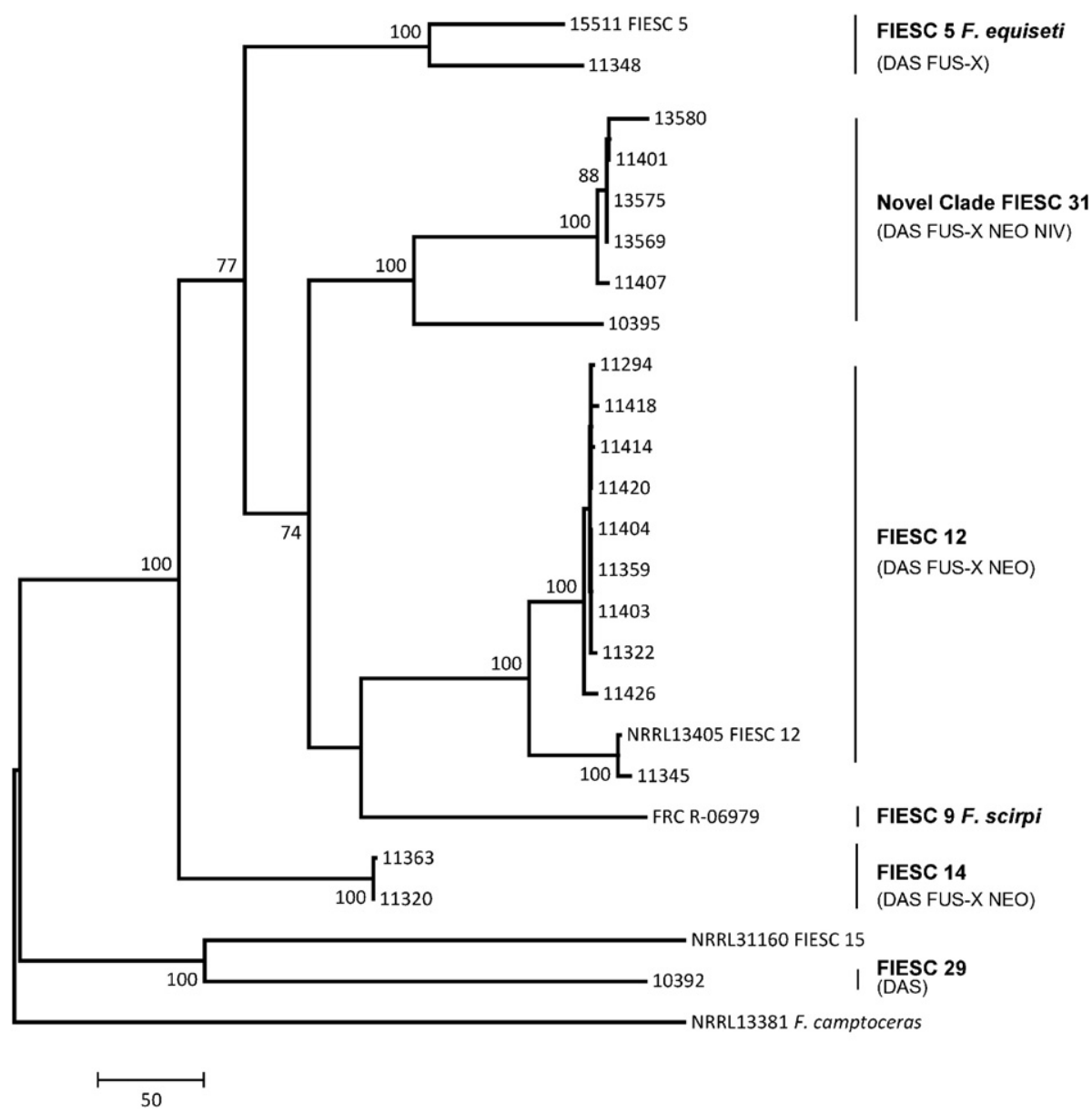

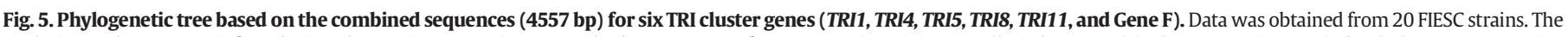

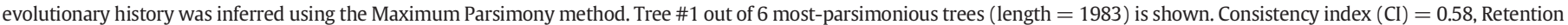
index $(\mathrm{RI})=0.79$. Numbers on branches indicate bootstrap values based on 500 pseudoreplicates.

Although members of FIESC are considered moderately aggressive on cereals, some of them have been reported to produce toxins, including both type A and type B trichothecenes. However, reports on trichothecene production in the FIESC present an inconsistent picture that suggests production is variable among and/or within species. For example, Kosiak et al. (2005) reported production of high levels of type A trichothecenes (e.g. DAS and NEO), significant levels of NIV and FUS-X (type B), but no DON or 3/15-ADON (type B) in an analysis of isolates identified as $F$. equiseti collected from Norwegian cereals. In contrast, Marın et al. (2012) reported production of DON as well as NIV, NEO and DAS in an analysis of $F$. equiseti strains collected from cereals from multiple locations around the world. These differences in mycotoxin profile could partly be attributed to the influence of the conditions under which the isolates were grown for toxin production/detection. In the study of Kosiak et al. (2005), isolates were grown for two weeks in darkness on YES medium, while wheat was used as substrate by Marın et al. (2012) and the cultures were incubated for four weeks under fluorescence light. The differences could also be due to the fact that most of $F$. equiseti isolates analyzed by Kosiak et al. (2005) belong to FIESC 14 (F. equiseti Type I, as reported by Castellá and Cabanes, 2014) population, while the study of Marın et al. (2012) included mainly FIESC 5 (F. equiseti Type II, as reported by Castella et al. 2014) F. equiseti isolates. Results reported in the current study (isolates were grown on PDA for two weeks) are more consistent with those of Kosiak et al. (2005) in that we detected production of DAS and NEO as well as the type B trichothecenes FUS-X and NIV, but we did not detect production of DON, 3-ADON, and 15-ADON. Interestingly, the majority of $F$. equiseti isolates included in our study originated in Canada and most probably belong to FIESC 14 (Type I) based on the study of Marın et al. (2012). This finding suggests that most of the Canadian isolates reported as F. equiseti are FIESC 14 (Type I), while FIESC 5 (Type II) includes mainly strains that occur in Southern Europe. All isolates (except ITEM 7547) that we examined for trichothecenes produced DAS, $68 \%$ of the isolates produced FUS-X, $58 \%$ produced NEO, and $10 \%$ produced NIV. About $30 \%$ of the isolates produced only type A (DAS/ NEO), while over $65 \%$ produced both type A (DAS/NEO) and B trichothecenes (FUS-X/NIV). NIV production was detected only in FIESC 31. Therefore, the results of our trichothecene production analysis provide further evidence that members of FIESC produce both type A and B trichothecenes. However, we found no evidence for production of 8aceylated type A trichothecenes such as T-2 toxin and HT-2 toxin. As far as we are aware, this is only the second report (Marın et al., 2012) that has combined an MS-based determination of trichothecene chemotype and phylogenetic-based determination of species identity of FIESC isolates.

Recent studies (Proctor et al., 2009; McCormick et al., 2013) reported that the synteny of several TRI genes within the TRI cluster in the FIESC is different than in other trichothecene-producing lineages of Fusarium, such as the well studied species $F$. graminearum and $F$. sporotrichioides. In the FIESC, TRI1 and TRI101 are located in the trichothecene cluster; TRI12 is absent; the TRI3-TRI7-TRI8 region of the cluster is in the opposite orientation and located at the opposite end of the cluster; and there is a transcription factor gene (Gene F) located between TRI5 and TRI6. It is not clear whether the modified arrangement of the trichothecene cluster is related to the variability in trichothecene production within the FIESC and to production of both type A and type B 
trichothecenes. However, variation in trichothecene production as well as production of both type A and B trichothecenes has also been reported in F. poae (Thrane et al., 2004), a species that is more closely related to and has a trichothecene cluster more similar to the cluster in $F$. graminearum and F. sporotrichioides (O'Donnell et al., 2013).

In the current study, the TRI-gene phylogeny was highly concordant with the phylogeny derived from housekeeping gene sequences. However, there was poor correlation between trichothecene production profile and phylogenetic relationships within the TRI-gene phylogeny (Fig. 5, Supplementary Table S1; Supplementary Figs. S3-S8). Furthermore, the TRI-gene phylogeny suggests that FIESC 31 isolates are more closely related to FIESC 12, while the housekeeping gene phylogeny suggests that FIESC 31 is more closely related to FIESC 9 (Figs. 1, 2). The lack of amplification products most likely resulted from a primer sequence issue or some other technical problem, because two strains that did not yield a TRI5 amplicon produced trichothecenes (ITEM 10391 and ITEM 11297), and yet TRI5 is essential for trichothecene production (Alexander et al., 2009).

The proteomic profiles obtained by MALDI-TOF MS corroborated the MLST analysis, in that different phylogenetic species were resolved into different clades in both analyses. However, the correlation was not perfectly concordant because some isolates in the same phylogenetic species (according to the DNA analysis) were resolved into different clades in the MALDI-TOF MS analysis. In particular, among isolates identified as FIESC 12, ITEM 11276, ITEM 11426 and ITEM 11404 formed a cluster that was more closely related to the FIESC 14 isolate ITEM 11320. Although the MALDI-TOF data were consistent with the DNAbased phylogenetic analysis with respect to resolving FIESC 31 isolates as an exclusive lineage, the two analyses differed in placement of FIESC 31 within the Equiseti clade. In the MALDI-TOF analysis, FIESC 31 isolates formed a sister group to all other isolates within the Equiseti clade, whereas multigene species phylogenies suggest that FIESC 31 was most closely related to FIESC 9 (F. scirpi) and FIESC 12 (Figs. 2, 3).

In conclusion, our polyphasic analysis of diversity of FIESC from cereal resolved five phylogenetic species, including a novel species, FIESC 31. The analysis has provided the first evidence for the occurrence of FIESC 12 in cereals, FIESC 29 in maize and rice, FIESC 14 in oat and maize, and FIESC 5 in oat, maize, rice, soil, and barley. Furthermore, the data provide evidence that trichothecene production is variable within the FIESC. Further studies are required to determine the genetic basis for this variability.

Supplementary data to this article can be found online at http://dx. doi.org/10.1016/j.ijfoodmicro.2016.06.023.

\section{Acknowledgment}

This work was supported by the EU project EC KBBE-2007-222690-2 MYCORED.

\section{References}

Alexander, N.J., Proctor, R.H., McCormick, S.P., 2009. Genes, gene clusters, and biosynthesis of trichothecenes and fumonisins in Fusarium. Toxin Rev. 28, 198-215.

Balmas, V., Migheli, Q., Scherm, B., Garau, P., O'Donnell, K., Ceccherelli, G., Kang, S., Geiser, D.M., 2010. Multilocus phylogenetics show high levels of endemic fusaria inhabiting Sardinian soils Tyrrhenian Islands. Mycologia 102, 803-812. http://dx.doi.org/10. 3852/09-201.

Brown, D.W., McCormick, S.P., Alexander, N.J., Proctor, R.H., Desjardins, A.E., 2001. A genetic and biochemical approach to study trichothecene diversity in Fusarium sporotrichioides and Fusarium graminearum. Fungal Genet. Biol. 32 (2), 121-133.

Castellá, G., Cabañes, F.J., 2014. Phylogenetic diversity of Fusarium incarnatum-equiseti species complex isolated from Spanish wheat. Antonie Van Leeuwenhoek 106 (2), 309-317.

Desjardins, A.E., 2006. Fusarium mycotoxins, chemistry, genetics, and biology. American Phytopathological Society APS Press, St. Paul Minnesota, USA.

European Commission, 2015. Agricultural and rural development, short-term outlook http://ec.europa.eu/agriculture/markets-and-prices/short-term-outlook/index_en. htm- (Accessed 10/07/2015).

FAOSTAT, 2013. Food and agriculture Organization of the United Nations, FAO statistical database. http://faostat.fao.org/site/291/default.aspx (Accessed 19/06/2013).
Lambotte, Fautrey, 1894. Rev. Mycol. (Toulouse) 16 (n. 63), 111.

Felsenstein, J., 1985. Confidence limits on phylogenies: An approach using the bootstrap. Evolution 39, 783-791

Geiser, D.M., del Mar Jiménez-Gasco, M., Kang, S., Makalowska, I., Veeraraghavan, N., Ward, T.J., Zhang, N., Kuldau, G.A., O'Donnell, K., 2004. FUSARIUM-ID v. 1.0: A DNA sequence database for identifying Fusarium. Eur. J. Plant Pathol. 110, 473-479.

Glass, N.L., Donaldson, G.C., 1995. Development of primer sets designed for use with the PCR to amplify conserved genes from filamentous ascomycetes. Int. J. Food Microbiol. 61, 1323-1330.

Grovey, J.F., 2007. The trichothecenes and their biosynthesis. In: Herz, W., Falk, H., Kirby, G.W. (Eds.), Progress in the Chemistry of Organic Natural Products. Springer-Verlag, Vienna, pp. 63-130.

Jurado, M., Vázquez, C., Patiño, B., González-Jaén, M.T., 2005. PCR detection assay for the trichothecene-producing species Fusarium graminearum, Fusarium culmorum, Fusarium poae, Fusarium equiseti and Fusarium sporotrichioides. Syst. Appl. Microbiol. 28, $562 \mathrm{e} 568$.

Jurado, M., Vázquez, C., Callejas, C., González-Jaén, M.T., 2006. Occurrence and variability of mycotoxigenic Fusarium species associated to wheat and maize in the south of Spain. Mycotoxin Res. 22, 87e91.

Kosiak, E.B., Holst-Jensen, A., Rundberget, T., Gonzalez-Jaen, M.T., Torp, M., 2005. Morphological, chemical and molecular differentiation of Fusarium equiseti isolated from Norwegian cereals. Int. J. Food Microbiol. 99, 195-206.

Kristensen, R., Torp, M., Kosiak, B., Holst-Jensen, A., 2005. Phylogeny and toxigenic potential is correlated in Fusarium species as revealed by partial translation elongation factor 1 alpha gene sequences. Mycol. Res. 109, 173-186. http://dx.doi.org/10.1017/ S0953756204002114.

Leslie, J.F., Summerell, B.A., 2006. Fusarium laboratory workshops, a recent history. Mycotoxin Res. 22, 73-74.

Liu, Y.L., Whelen, S., Hall, B.D., 1999. Phylogenetic relationships among ascomycetes, evidence from an RNA polymerase II subunit. Mol. Bio. Evol. Soc. 16, 1799-1808.

Ma, L.J., Geiser, D.M., Proctor, R.H., Rooney, A.P., O'Donnell, K., Trail, F., Gardiner, D.M., Manners, J.M., Kazan, K., 2013. Fusarium Pathogenomics. Annu. Rev. Microbiol. 67, 399-416.

Maier, F.J., Miedaner, T., Hadeler, B., Felk, A., Salomon, S., Lemmens, M., Kassner, H. Schäfer, W., 2006. Involvement of trichothecenes in fusarioses of wheat, barley and maize evaluated by gene disruption of the trichodiene synthase (Tri5) gene in three field isolates of different chemotype and virulence. Mol. Plant Pathol. 7, 449-461.

Marın, P., Moretti, A., Ritieni, A., Jurado, M., Vazquez, C., Gonzalez, J.M.T., 2012. Phylogenetic analysis and toxigenic profiles of Fusarium equiseti and Fusarium acuminatum isolated from cereals from southern Europe. Food Microbiol. 31, 229-237.

Marinach-Patrice, C., Lethuillier, A., Marly, A., Brossas, J.Y., Gene, J., Symoens, F., Datry, A. Guarro, J., Mazier, D., Hennequin, C., 2009. Use of mass spectrometry to identify clinical Fusarium isolates. Clin. Microbiol. Infect. 15, 634-642.

McCormick, S.P., 2013. Microbial detoxification of mycotoxins. J. Chem. Ecol. 39, 907-918.

McCormick, S.P., Alexander, N.J., Proctor, R.H., 2013. Trichothecene triangle: toxins, genes, and plant disease. Phytochemicals, Plant Growth, and the Environment. Springer, pp. 1-17.

Moretti, A., Logrieco, A., Bottalico, A., Ritieni, A., Fogliano, V., Randazzo, G., 1996. Diversity in beauvericin and fusaproliferin production by different populations of Gibberella fujikuroi Fusarium section Liseola. Sydowia 48, 44-56.

Moretti, A., Susca, A., Mulé, G., Logrieco, A.F., Proctor, R.H., 2013. Molecular biodiversity of mycotoxigenic fungi that threaten food safety. Int. J. Food Microbiol. 167, 57-66.

O'Donnell, K., Cigelnik, E., Nirenberg, H.I., 1998. Molecular systematics and phylogeography of the Gibberella fujikuroi species complex. Mycologia 90, 465-493.

O'Donnell, K., Sarver, B.A., Brandt, M., Chang, D.C., Noble-Wang, J., Park, B.J., Sutton, D.A., Benjamin, L., Lindsley, M., Padhye, A., Geiser, D.M., Ward, T.J., 2007. Phylogenetic diversity and microsphere array-based genotyping of human pathogenic fusaria, including isolates from the multistate contact lens-associated US keratitis outbreaks of 2005 and 2006. J. Clin. Microbiol. 45 (7), 2235-2248.

O'Donnell, K., Sutton, D.A., Rinaldi, M.G., Gueidan, C., Crous, P.W., Geiser, D.M., 2009. A novel multi-locus sequence typing scheme reveals high genetic diversity of human pathogenic members of the Fusarium incarnatum-F. equiseti and F. chlamydosporum species complexes within the U.S. J. Clin. Microbiol. 47, 3851-3861.

O'Donnell, K., Sutton, D.A., Rinaldi, M.G., Sarver, B.A.J., Balajee, S.A., Schroers, H.J., Summerbell, R.C., Robert, V.A.R., Crous, P.W., Zhang, N., Aoki, T., Jung, K., Park, J. Lee, Y.H., Kang, S., Park, B., Geiser, D.M., 2010. Internet-accessible DNA sequence database for identifying fusaria from human and animal infections. J. Clin. Microbiol. 48, $3708 \mathrm{e} 3718$.

O'Donnell, K., Humber, R. A., Geiser, D.M., Kang S., Park, B., Robert, V. A. Crous, P.W. Johnston, P.R., Aoki, T., Rooney, A.P., Rehner, S.A., 2012. Phylogenetic diversity of insecticolous fusaria inferred from multilocus DNA sequence data and their molecular identification via FUSARIUM-ID and FUSARIUM MLST. Mycologia 104, 427 e445.

O'Donnell, K., Nirenberg, H.I., Aoki, T., Cigelnik, E., 2000. A multigene phylogeny of the Gibberella fujikuroi species complex, detection of additional phylogenetically distinct species. Mycoscience 41, 61-78.

O'Donnell, K., Rooney, A.P., Proctor, R.H., Brown, D.W., McCormick, S.P., Ward, T.J., Frandsen, R.J., Lysøe, E., Rehner, S.A., Aoki, T., Robert, V.A., Crous, P.W., Groenewald, J.Z., Kang, S., Geiser, D.M., 2013. Phylogenetic analyses of RPB1 and RPB2 support a middle cretaceous origin for a clade comprising all agriculturally and medically important Fusaria. Fungal Genet. Biol. 52, 20-31.

Proctor, R.H., Brown, D.W., Plattner, R.D., Desjardins, A.E., 2003. Co-expression of 15 contiguous genes delineates a fumonisin biosynthetic gene cluster in Gibberella moniliformis. Fungal Genet. Biol. 38, 237-249. 
Proctor, R.H., McCormick, S.P., Alexander, N.J., Desjardins, A.E., 2009. Evidence that a secondary metabolic biosynthetic gene cluster has grown by gene relocation during evolution of the filamentous fungus Fusarium. Mol. Microbiol. 74, 1128-1142.

Ranque, S., Normand, A.C., Cassagne, C., Murat, J.B., Bourgeois, N., Dalle, F., Gari-Toussaint, M., Fourquet, P., Hendrickx, M., Piarroux, R., 2014. MALDI-TOF mass spectrometry identification of filamentous fungi in the clinical laboratory. Mycoses 57, 135-140. http://dx.doi.org/10.1111/myc.12115.

Rocha, O., Ansari, K., Doohan, F.M., 2005. Effects of trichothecene mycotoxins on eukaryotic cells, a review. Food Addit. Contam. 22 (4), 369-378.

Rodrigues, P., Santos, C., Venâncio, A., Lima, N., 2011. Species identification of Aspergillus section Flavi isolates from Portuguese almonds using phenotypic, including MALDITOF ICMS, and molecular approaches. J. Appl. Microbiol. 111, 877-892.

Saccardo, P.A., 1886. Sylloge Hyphomycetum. Sylloge Fungorum 4, 1-807.

Santos, C., Ventura, J.A., Costa, H., Fernandes, P.M.B., Lima, N., 2015. MALDI-TOF MS to identify the pineapple pathogen Fusarium guttiforme and its antagonist Trichoderma asperellum on decayed pineapple. Trop. Plant Pathol. http://dx.doi.org/10.1007/ s40858-015-0027-7 (On-line).

Short, D.P.G., O'Donnell, K., Zhang, N., Juba, J.H., Geiser, D.M., 2011. Widespread occurrence of diverse human pathogenic types of the fungus Fusarium detected in plumbing drains. J. Clin. Microbiol. 49, 4264-4272.

Silva, F.C., Chalfoun, S.M., Batista, L.R., Santos, C., Lima, N., 2015. Use of a polyphasic approach including MALDI-TOF MS for identification of Aspergillus section Flavi strain isolated from food commodities in Brazil. Ann. Microbiol. http://dx.doi.org/10.1007/ s13213-015-531 1050-0 (on-line).
Susca, A., Mulè, G., Mastropasqua, L., Paciolla, C., 2015. Biodiversità molecolare di Fusarium semitectum isolati da Hylocereus undatus e altri ospiti. Inf. Bot. Ital. 47 (2), 291-313.

Tamura, K., Peterson, D., Peterson, N., Stecher, G., Nei, M., Kumar, S., 2011. MEGA5, molecular evolutionary genetics analysis using maximum likelihood, evolutionary distance, and maximum parsimony methods. Mol. Biol. Evol. 28, 2731-2739.

Thompson, J.D., Higgins, D.G., Gibson, T.J., 1994. CLUSTAL 541 W, improving the sensitivity of progressive multiple sequence alignment through sequence weighting, positionspecific gap penalties and weight matrix choice. Nucleic Acids Res. 22, 4673-4680.

Thrane, U., Adler, A., Clasen, P., Galvano, F., Langseth, W., Lew, H., Logrieco, A., Nielsen, K.F., Ritieni, A., 2004. Diversity in metabolite production by Fusarium langsethiae, Fusarium poae, and Fusarium sporotrichioides. Int. J. Food Microbiol. 95, 257-266.

van Diepeningen, A.D., Brankovics, B., Iltes, J., van der Lee, T.A.J., Waalwijk, C., 2015. Diagnosis of Fusarium infections, approaches to identification by the clinical mycology laboratory. Curr. Fungal Infect. Rep. 9, 135-143.

Ward, T.J., Bielawski, J.P., Kistler, H.C., Sullivan, E., O'Donnell, K., 2002. Ancestral polymorphism and adaptive evolution in the trichothecene mycotoxin gene cluster of phytopathogenic Fusarium. Proc. Natl. Acad. Sci. 99, 9278-9283.

Watanabe, M., Yonezawa, T., Sugita-Konishi, Y., Kamata, Y., 2013. Utility of the phylotoxigenic relationships among trichothecene-producing Fusarium species for predicting their mycotoxin-producing potential. Food Addit. Contam. A 30 (8), 1370-1381. 